\title{
Estudio general del método de síntesis de pirólisis de aerosol en Ilama
}

\author{
General study of Flame Spray \\ Pyrolysis synthesis method
}

Natalia Betancur-Granados ${ }^{1,2}$, Jorge I. Tobón ${ }^{2}$, Oscar Jaime Restrepo-Baena ${ }^{2}$

\footnotetext{
${ }^{1}$ Facultad de Ingeniería, Grupo Innovación e Ingeniería I², Corporación Universitaria Minuto de Dios - UNIMINUTO, Cra. 45 No. 22D - 25 - Km 0 Autopista Medellín - Bogotá, 051052 Medellín, Colombia.

${ }^{2}$ Departamento de Materiales y Minerales. Grupo de Investigación del Cemento y Materiales de Construcción (CEMTCO), Universidad Nacional de Colombia, Calle 65 No 59 A-110, Medellín 050036, Colombia.

e-mail: natalia.betancur.g@uniminuto.edu.co, jitobon@unal.edu.co, ojrestre@unal.edu.co
}

\section{RESUMEN}

Pirólisis de aerosol en llama (FSP), es una de las metodologías más prometedoras en la producción de nanopartículas a gran escala, dado su potencial de escalamiento y producción en continuo. Este artículo aborda los fenómenos químicos y físicos ocurridos durante el proceso, desde la preparación de la solución de precursores, los procesos de transformación en la llama y la formación de nanopartículas, los cuales permiten explicar las propiedades de los productos de reacción. Finalmente, se presenta el equipo de síntesis, sus componentes y la importancia del diseño del equipo sobre las propiedades de los materiales.

Palabras clave: Pirólisis de aerosol en llama, pirólisis por pulverización de llama, pirólisis por rociado de llama, Síntesis alternativa, aerosol.

\section{ABSTRACT}

Flame Spray Pyrolysis (FSP) is one of the most promising methodologies in large-scale nanoparticle production, given its potential for scaling and continuous production. This review addresses the chemical and physical phenomena that occur during the process, from the preparation of the starting solution, the transformation processes in the flame and the formation of nanoparticles, allowing to explain the properties of the products. Finally, the synthesis setup, its components, and the importance its design on the properties of materials are presented.

Keywords: Flame Spray Pyrolysis, Liquid-feed flame spray pyrolysis, In-flame-reaction, Alternative synthesis, Spray.

\section{INTRODUCCIÓN}

Los procesos de aerosol en llama son desarrollados por M. Sokolowski en 1977 al sintetizar nanopartículas de $\mathrm{Al}_{2} \mathrm{O}_{3}$, en un proceso continuo que denominó Reacción en llama [1]. Esta metodología fue poco aplicada, hasta ser desarrollada durante los años 90's en la Universidad de Michigan, por el Centro de Ciencia macromolecular e Ingeniería liderados por el profesor Richard Laine, y de manera simultánea en el Instituto Federal Suizo de Tecnología (ETH Zurich), acuñando el término de Pirólisis de aerosol en llama (FSP, Flame Spray Pyrolysis) [2-5].

El término pirólisis, generalmente se refiere a la descomposición química a altas temperaturas en ausencia de oxígeno, sin embargo, en pirólisis de aerosol en llama (FSP) sólo hace referencia a procesos a alta temperatura, durante los cuales se forman óxidos complejos a partir de una solución estequiométrica de precursores cerámicos [6], en un proceso continuo de alta eficiencia y alto potencial de industrialización, donde se dan reacciones de descomposición y oxidación, cuando un aerosol entra en contacto con una llama de combustión [7], dando lugar a la formación de nanopartículas.

El uso industrial de nanopartículas se ha extendido rápidamente, por lo cual, migrar de procesos quími- 
cos en lotes a procesos de aerosol en continuo, sumado al entendimiento de los procesos de formación de nanopartículas mediante FSP, permite avanzar hacia la fabricación de nanopartículas a gran escala con aplicaciones industriales en diferentes campos [8, 9]. Por tanto, en este artículo, se hace una revisión de los fenómenos involucrados durante el proceso de Pirólisis de aerosol en llama, condiciones de síntesis, partes que componen el equipo y un breve acercamiento hacia el futuro del proceso. La Tabla 1 muestra la nomenclatura de las variables involucradas durante las ecuaciones que modelan los procesos de pirólisis de aerosol en llama.

Tabla 1: Nomenclatura de las variables involucradas en los procesos de pirólisis de aerosol en llama.

\begin{tabular}{|c|c|}
\hline Variable & Nombre \\
\hline$a_{0}$ & Área superficial del monómero \\
\hline$a_{s}$ & Área superficial de un agregado fundido \\
\hline $\mathrm{B}_{0, \mathrm{q}}$ & Número de transferencia \\
\hline $\mathrm{C}$ & Concentración del soluto \\
\hline $\mathrm{c}$ & Velocidad de la partícula \\
\hline $\mathrm{Cu}$ & Factor de corrección de Cunningham \\
\hline $\mathrm{C}_{0}$ & Concentración de equilibrio \\
\hline $\mathrm{C}_{\mathrm{D}}$ & Concentración de la gota \\
\hline $\mathrm{C}_{\mathrm{NS}}$ & Coeficiente de arrastre en el régimen no laminar \\
\hline $\mathrm{D}$ & Coeficiente de difusión de la partícula \\
\hline $\mathrm{d}_{\mathrm{d}}$ & Diámetro de la gota \\
\hline $\mathrm{D}_{\mathrm{f}}$ & Dimensión similar a un fractal de masa \\
\hline $\mathrm{dN} / \mathrm{dt}$ & Evolución de la distribución del tamaño de partícula de los agregados \\
\hline $\mathrm{d}_{\mathrm{p}}$ & Diámetro de partícula \\
\hline $\mathrm{E}$ & Campo eléctrico del colector \\
\hline ESP & Precipitador electrostático \\
\hline FSP & Pirólisis de aerosol en llama \\
\hline g & Parámetro de transición \\
\hline $\mathrm{K}$ & Constante de Boltzmann \\
\hline MW & Peso molecular de las partículas \\
\hline $\mathrm{N}$ & Número de partículas por área de volumen de gas \\
\hline $\mathrm{n}$ & Relación estequiométrica \\
\hline $\mathrm{n}_{\mathrm{p}}$ & Número de partículas en un agregado \\
\hline $\mathrm{Nu}$ & Número de Nusselt \\
\hline $\mathrm{P}$ & Probabilidad de una fluctuación termodinámica de energía libre crítica \\
\hline $\mathrm{Q}_{\mathrm{A}}$ & Tasa de flujo volumétrico del aire \\
\hline $\mathrm{Q}_{\mathrm{L}}$ & Tasa de flujo volumétrico del líquido \\
\hline Q & Caudal volumétrico de gas \\
\hline q & Carga de la partícula \\
\hline $\mathrm{r}$ & Radio de núcleo esférico \\
\hline $\mathrm{r}^{*}$ & Radio crítico \\
\hline$r_{c}$ & Radio de colisión \\
\hline $\operatorname{Re}$ & Número de Reynolds \\
\hline $\mathrm{R}_{\mathrm{N}}$ & Tasa de nucleación por volumen y unidad de tiempo \\
\hline $\mathrm{r}_{\mathrm{p}}$ & Radio de la partícula principal \\
\hline SMD & Diámetro medio de Sauter \\
\hline
\end{tabular}




\begin{tabular}{|c|c|}
\hline$\beta$ & Velocidad de colisión \\
\hline $\mathrm{T}$ & Temperatura \\
\hline $\mathrm{t}$ & Tiempo para el precalentamiento a presión atmosférica \\
\hline $\mathrm{T}_{\mathrm{bp}(\text { disolvente) }}$ & Temperatura de ebullición del disolvente \\
\hline $\mathrm{T}_{\mathrm{d} / \mathrm{p} \text { (precursores) }}$ & Temperatura de descomposición de los precursores \\
\hline $\mathrm{u}_{\mathrm{f}}$ & Velocidad del gas \\
\hline $\mathrm{U}_{\mathrm{R}}$ & Velocidad relativa de aire al líquido \\
\hline$V$ & Velocidad de la llama \\
\hline$v$ & Volumen agregado \\
\hline$v_{0}$ & Volumen del monómero \\
\hline$v_{d}$ & Velocidad de la gota \\
\hline$v_{p}$ & Volumen de las partículas primarias \\
\hline$v_{\text {rel }}$ & Velocidad relativa del gas y la gota \\
\hline$\Gamma$ & Frecuencia de salto de las especies en crecimiento \\
\hline$\Delta \mu_{\mathrm{s}}$ & Energía superficial de la nueva fase sólida \\
\hline$\Delta \mu_{\mathrm{v}}$ & Cambio en el volumen de energía \\
\hline$\Delta \mathrm{G}^{*}$ & Energía libre de Gibbs crítica \\
\hline$\Delta \mathrm{G}_{V}$ & Energía libre de Gibbs \\
\hline$\lambda$ & Diámetro de las especies en crecimiento \\
\hline$\rho$ & Densidad de las partículas \\
\hline$\rho_{\mathrm{L}}$ & Densidad del líquido \\
\hline$\sigma$ & Sobresaturación \\
\hline$\overline{\sigma_{\mathrm{L}}}$ & Tensión superficial del líquido \\
\hline$\tau$ & Tiempo de sinterización \\
\hline $\mathrm{r}$ & Energía superficial por unidad de área \\
\hline$\Omega$ & Volumen atómico \\
\hline$\mu_{\mathrm{L}}$ & Viscosidad del líquido \\
\hline$\mu$ & Viscosidad de partícula \\
\hline$\eta$ & Eficiencia de recolección del precipitador \\
\hline Ac & Área de recolección efectiva \\
\hline Vpm & Velocidad de migración de partículas \\
\hline
\end{tabular}

\section{PIRÓLISIS DE AEROSOL EN LLAMA}

En pirólisis de aerosol en llama, una solución líquida de precursores, es dispersada por una corriente de gas, resultando en la formación de un aerosol el cual es evaporado al atravesar una llama con temperatura superior a $1200{ }^{\circ} \mathrm{C}$ [10-12]. La fase gaseosa resultante, reacciona y se transforma en partículas primarias, las cuales sufren procesos de crecimiento hasta su recolección como agregados de nanopartículas, como se explicará en las siguientes secciones. Los parámetros operacionales como flujos de alimentación, dispersión, precursores, concentraciones, entre otros, y las especificaciones del equipo de síntesis, como dimensiones de la boquilla y los quemadores, determinan las propiedades de las nanopartículas, por lo cual su correcta manipulación y entendimiento supone un reto para los investigadores que estudian este proceso [13].

\subsection{Precursores y preparación de la solución de partida}

Una gran variedad de compuestos orgánicos e inorgánicos pueden ser usados como solutos y disolventes para el proceso. Los solutos más utilizados son acetatos, nitratos, organometálicos y metalorgánicos [14, 15]. Las sales inorgánicas como nitratos poseen un bajo costo y alta solubilidad en disolventes polares, sin embargo suelen producir morfologías no esféricas y micropartículas [14, 16, 17]. Por otra parte, los compuestos meta- 
lorgánicos y organometálicos son estables en solución y favorecen la formación de nanopartículas [18]. Para determinar la cantidad de precursor cerámico que puede ser transformado en el producto final, se usa el concepto de rendimiento cerámico, el cual es definido como la "masa de cerámico expresada como porcentaje de la masa del precursor cerámico usado durante el proceso de ceramización" [6], el cual puede ser hallado experimentalmente mediante un análisis termogravimétrico o un análisis de pérdida de peso por calcinación del material, y teóricamente, mediante el rendimiento cerámico teórico [6].

La concentración de la solución depende de la solubilidad y viscosidad del precursor. Soluciones viscosas impiden una buena formación del aerosol durante el proceso, mientras que precursores poco solubles requieren la adición de agentes quelantes, dificultando la preparación de la solución [19].

El tamaño y la morfología de las partículas son afectados por la concentración de la solución, definida mediante el término carga cerámica, el cual es la relación entre la masa del producto y la masa del disolvente (Ecuación 1). La literatura sugiere cargas cerámicas inferiores al $10 \%$ para obtener nanopartículas esféricas, ya que concentraciones superiores pueden formar partículas huecas y grandes [12, 19-21].

$$
\text { Carga cerámica }=100 \% \times \frac{\text { masa del producto }}{\text { masa del disolvente }}
$$

La elección de los disolventes depende de la solubilidad de los precursores, la entalpía de vaporización y el costo. El disolvente más utilizado es el etanol, y en menor medida metanol, tetrahidrofurano y xileno [19]. Su elección puede basarse en la relación entre la temperatura de ebullición del disolvente $\left(\mathrm{T}_{\mathrm{bp}}\left(\right.\right.$ disolvente)) y la temperatura de descomposición de los precursores ( $\mathrm{T}_{\mathrm{d} / \mathrm{p}}($ precursores $)$ ), $\mathrm{T}_{\mathrm{bp}}$ (disolvente)/ $\mathrm{T}_{\mathrm{d} / \mathrm{p}}$ (precursores), determinando el mecanismo de formación de las partículas. En una solución donde el disolvente posee un punto de ebullición mayor a la temperatura de descomposición de los precursores, $\left(\mathrm{T}_{\mathrm{bp}} / \mathrm{T}_{\mathrm{d} / \mathrm{p}}>1,05\right)$, los precursores metálicos se evaporan, reaccionan en fase gaseosa y forman nanopartículas. En contraste, al usar disolventes de bajo punto de ebullición $\left(\mathrm{T}_{\mathrm{bp}} / \mathrm{T}_{\mathrm{d} / \mathrm{p}}<1,05\right)$, el disolvente se evapora antes que los precursores, precipitandose y formando partículas grandes y/o huecas (Figura 1) [19, 22]. Otro criterio para elegir el disolvente es la entalpía de combustión, donde entalpías superiores a 4,7 KJ/g son suficientes para evaporar los precursores y formar nanopartículas, incluso si $\mathrm{T}_{\mathrm{bp}} / \mathrm{T}_{\mathrm{d} / \mathrm{p}}<1.05[19,23]$.
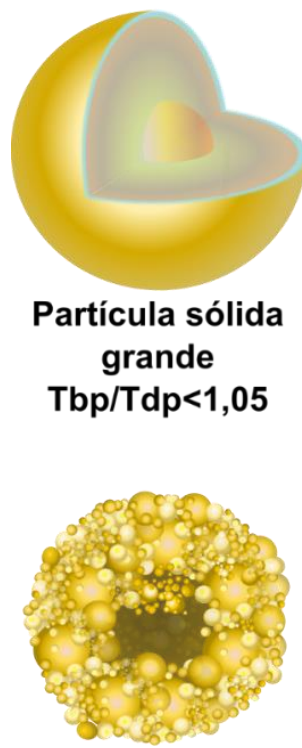

Fusión de micro y nanopartículas
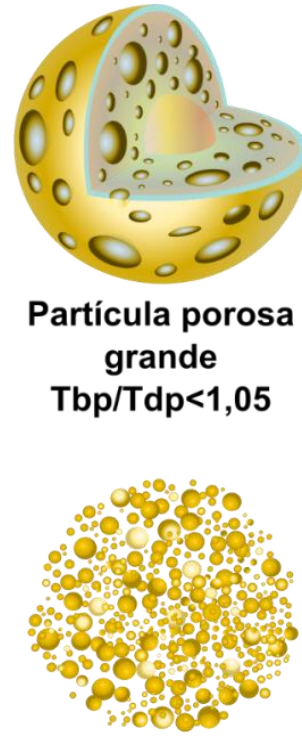

Nanopartículas

Tbp/Tdp $>1,05$

Figura 1: Partículas formadas mediante FSP

\subsection{Mecanismo de formación de las partículas}

La formación de partículas en pirólisis de aerosol en llama puede estudiarse como un proceso bottom-up, caracterizado por la formación de partículas con morfología uniforme, constituidas molécula por molécula $[17,24]$. En este enfoque, las partículas se forman a partir de una solución supersaturada seguida de procesos 
de nucleación y crecimiento regidos por el equilibrio termodinámico [24].

En la llama pueden darse dos mecanismos de formación de las partículas, correspondientes a mecanismo gota-partícula que conduce a la formación de partículas grandes o huecas, y gas-partícula que conduce a la formación de nanopartículas, teniendo como etapas la evaporación/descomposición de la solución, reacción en fase gaseosa, nucleación, coagulación y coalescencia (Figura 2) [25, 13, 26].

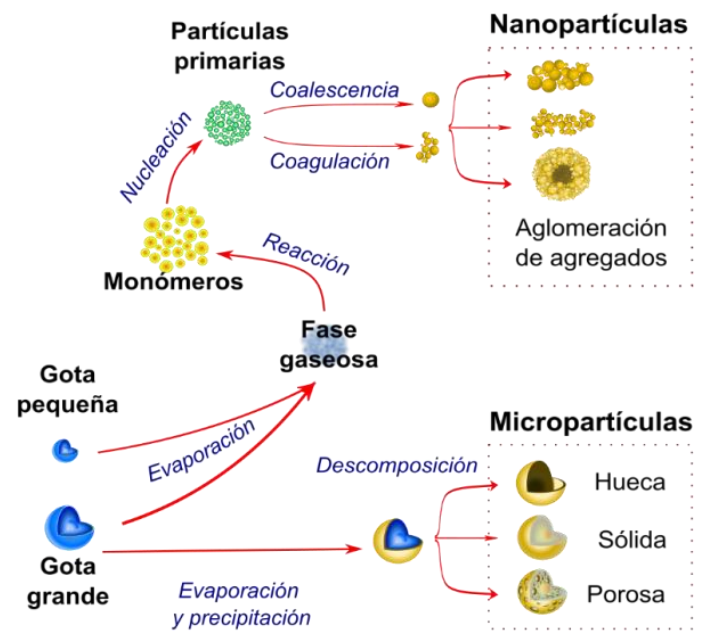

Figura 2: Mecanismo de formación de partículas en FSP

\subsubsection{Evaporación, descomposición y reacción}

La evaporación, como primera etapa de formación, determina el mecanismo de conversión de las gotas en partículas, ya sea gas-partícula ó gota-partícula.

Los fenómenos de transferencia de calor comienzan cuando las gotitas atraviesan la llama, se evapora el disolvente y reaccionan las especies químicas [9]. Las gotitas del aerosol podrían cruzar la llama antes de evaporarse completamente, dando lugar a la evaporación/precipitación por el gas caliente circundante. Luego, las fases gaseosas resultantes dan lugar a la nucleación y crecimiento de nanopartículas [24].

En pirólisis de aerosol en llama, el modelo de Sirignano (1999) describe la combustión de las gotas, considerando el equilibrio líquido-vapor cuando las gotitas están en contacto con combustibles y especies oxidantes. Este modelo supone que las fases gaseosas están formadas por el combustible, el oxidante y los productos de combustión, sin considerar la solubilidad del combustible líquido con los gases e interacciones entre las gotas. Además, supone difusividad de masa y conductividad térmica de los productos constantes, y reacción instantánea entre el combustible y el oxidante, permitiendo el cálculo de la velocidad de la gota, $v_{d}$ (2) y su diámetro, $d_{d}(3)$, en términos del número de Nusselt (4), número de transferencia, $\mathrm{B}_{0, \mathrm{q}}(5)$, número de Reynolds, $\operatorname{Re}(6)$ y el coeficiente de arrastre en el régimen no laminar $\mathrm{C}_{\mathrm{NS}}$ (8) [27]

$$
\begin{aligned}
& \frac{d v_{d}}{d x}=\frac{3}{4} \frac{\rho_{g}}{\rho_{d}} \frac{C_{N S}}{d_{d}}\left(v_{r e l}\right)\left|v_{\text {rel }}\right| \frac{1}{v_{d}} \\
& \frac{d d_{d}}{d x}=-\left(\frac{8 k_{g}}{\rho_{l} c_{p g}} \ln \left(1+B_{0, q}\right) \frac{N u}{2}\right) \frac{1}{d_{d}} \frac{1}{v_{d}} \\
& N u=2+\frac{0.555 \operatorname{Re}^{1 / 2} P r^{1 / 3}}{\left[1+1.232 /\left(\operatorname{RePr}^{4 / 3)}\right]^{1 / 2}\right.} \\
& B_{0, q}=\frac{\frac{\Delta h_{c}}{v}+c_{p g}\left(T_{\infty}-T_{d}\right)}{q_{i-l}+h_{i g}} \\
& R e=\frac{d_{d} v_{r e l}}{\eta} \\
& v_{r e l}=u_{f}-v_{d}
\end{aligned}
$$




$$
C_{N S}=\frac{24}{R e}+\frac{6}{1+\sqrt{R e}}+0.4
$$

En llamas de combustión poco energéticas, la velocidad de evaporación y reacción de los componentes de la solución varía. En caso contrario, los precursores se descomponen formando una fase gaseosa de radicales, intermediarios y monómeros que pueden polimerizar y crear núcleos. La cinética de las reacciones de combustión está débilmente acoplada a los procesos de descomposición de precursores y de formación de partículas; sin embargo, pueden darse interacciones entre ellos mediante reacciones de radicales.

Debido a que el tiempo de residencia de las especies en la llama es de microsegundos, la reacción química y formación de nanopartículas se supone instantánea [28]. Fristrom y Westenberg (1965), propusieron una relación empírica con las llamas premezcladas (9), donde el tiempo requerido $(t)$ a presión atmosférica, para el precalentamiento y la reacción química en la llama es inversamente proporcional al cuadrado del velocidad de la llama $(V)[28]$.

$$
t=\frac{3}{V^{2}}
$$

Los núcleos termodinámicos estables continuarán el proceso de nucleación [29]. Sin embargo, en soluciones de partida formadas por más de un precursor (A y B), si la velocidad de descomposición del componente A es muy superior a la velocidad de descomposición del componente $B$, se pueden formar partículas de composición heterogénea por la deposición de óxidos simples del componente $B$ sobre óxidos simples del componente $A$ [29].

\subsubsection{Nucleación}

El crecimiento de las nanopartículas supone una nucleación homogénea. Con el medio enriquecido en radicales, intermediarios y monómeros, la energía libre de Gibbs incrementa, permitiendo la polimerización y formación de núcleos para reducir la energía total del sistema, mediante la formación de una nueva fase. Por tanto, la fuerza impulsora para la nucleación y el crecimiento de las partículas es la reducción de la energía libre de Gibbs (ecuación 10) [24],

$$
\Delta G_{v}=\frac{-K T}{\Omega} \ln C / C_{0}=\frac{-K T}{\Omega} \ln (1-\sigma)
$$

En función de la concentración del soluto $(\mathrm{C})$, la concentración de equilibrio $\left(\mathrm{C}_{0}\right)$, la temperatura $(\mathrm{T})$, la constante de Boltzmann $(\mathrm{K})$, el volumen atómico $(\Omega)$ y la sobresaturación $(\sigma)$ descrita en la ecuación $(11)$,

$$
\sigma=\frac{C-C_{0}}{C_{0}}
$$

Considerando un núcleo esférico con radio $r$, el cambio en el volumen de energía, $\Delta \mu_{\mathrm{v}}(12)$ es,

$$
\Delta \mu_{v}=\frac{4}{3} \pi r^{3} \Delta G_{v}
$$

Al disminuir la sobresaturación, se reduce la energía, lo que se contrarresta con la introducción de la energía superficial de la nueva fase sólida. Esta energía se expresa como en la ecuación (13),

$$
\Delta \mu_{s}=4 \pi r^{2} \gamma
$$

Donde, $\gamma$ es la energía superficial por unidad de área.

El cambio total del potencial químico para la formación del núcleo es la suma de las ecuaciones (12) y (13). La reducción de energía dará como resultado la formación de partículas primarias si el radio excede un tamaño crítico, $r^{*}(14)$ y la energía crítica (15), continuando el proceso de nucleación y crecimiento, sino, la partícula se disolverá en el medio [24, 30].

$$
r^{*}=-\frac{2 \gamma}{\Delta \mathrm{G}_{v}}
$$




$$
\Delta G^{*}=\frac{16 \pi \gamma}{\left(3 \Delta G_{v}\right)^{2}}
$$

Finalmente, la tasa de nucleación por volumen y unidad de tiempo, $\mathrm{R}_{\mathrm{N}}$, para nucleación homogénea se estima mediante la ecuación (16),

$$
R_{N}=C_{0} \frac{K T}{3 \pi \lambda^{3} \mu_{L}} \exp \left(\frac{-\Delta G^{*}}{K T}\right)
$$

Donde, $\mathrm{C}_{0}$ es la concentración inicial del soluto, $\lambda$ es el diámetro de las especies en crecimiento y $\mu_{\mathrm{L}}$ es la viscosidad de la solución.

\subsubsection{Crecimiento superficial}

Los principales mecanismos de crecimiento de partículas en FSP son la coagulación, también llamada aglomeración, referida a la colisión de partículas que se unen entre sí, y la coalescencia, también llamada sinterización, referida a la fusión de coágulos para formar una partícula de tamaño mayor [31, 32]. Estos fenómenos son difíciles de separar, porque ambos ocurren al mismo tiempo.

Ulrich (1971) estudió los procesos de sinterización considerando la fusión y la coagulación individualmente, formulando ecuaciones para el tamaño de partícula y la evolución del área superficial específica [28, 33]. Kock y Frielander (1990) modelaron la evolución del tamaño de partícula primaria, considerando los efectos de la coagulación sobre los procesos de sinterización, suponiendo que la colisión de los agregados ocurre a la misma velocidad que las partículas primarias con el mismo volumen [34]. Xong y Pratsinis (1993), desarrollaron un modelo en dos dimensiones para una molécula libre en el régimen continuo, que describe la evolución del volumen agregado mediante coagulación y sinterización simultáneas en condiciones isotérmicas, asumiendo un agregado monodispersado y sin suposiciones sobre el tamaño de partícula [26, 35].

Después de la reacción química, las partículas primarias comienzan a crecer por colisiones sucesivas. Inicialmente, la tasa de coalescencia de las partículas es mayor que la tasa de colisión, lo que permite la formación de partículas esféricas. Suponiendo que todos los agregados tienen el mismo número de partículas primarias con el mismo tamaño, la evolución de la distribución del tamaño de partícula de los agregados se representa mediante la ecuación (17).

$$
\frac{d N}{d t}=-\frac{1}{2} \beta N^{2}
$$

Donde $\beta$, es la velocidad de colisión y $\mathrm{N}$ el número de partículas por área de volumen de gas. La ecuación (18) muestra como el área superficial de los agregados aumenta por coagulación y disminuye por sinterización,

$$
\frac{d a}{d t}=-\frac{1}{N} \frac{d N}{d t} a-\frac{1}{\tau}\left(a-a_{s}\right)
$$

Donde, $\tau$ es el tiempo de sinterización necesario para reducir aproximadamente el $63 \%$ del área superficial excedente de un agregado, sobre el área superficial de una esfera con la misma masa y $a_{s}$ el área superficial de un agregado completamente fundido en forma esférica (ecuación 19).

$$
a_{s}=\left(\frac{v}{v_{0}}\right)^{2 / 3} a_{0}
$$

Donde $a_{0}$ corresponde al área de superficie del monómero, $v_{0}$ el volumen del monómero y $v$ el volumen agregado, que solo se ve afectado por la coagulación. Además, el tamaño de partícula $\left(d_{p}\right)$ y el número de partículas $\left(n_{p}\right)$ en un agregado se describen en las ecuaciones $(20)$ y (21), donde $v_{p}=\left(\pi d_{p}^{3}\right) / 6$ es el volumen de las partículas primarias.

$$
\begin{aligned}
& d_{p}=\frac{6 v}{a} \\
& n_{p}=\frac{v}{v_{p}}
\end{aligned}
$$


La coagulación ocurre entre partículas primarias adyacentes, lo que reduce el número de partículas en los agregados. La morfología de las partículas está determinada por la velocidad de coagulación, $\beta$, y el tiempo de sinterización característico $\tau$ [35]. Un modelo aceptado para la coagulación es el modelo de colisión de Kernel, en el cual los agregados de partículas se someten a procesos de alta temperatura y se tratan como fractales, utilizando una relación entre el volumen agregado y el radio de giro o radio de colisión [35]. El concepto similar a un fractal, para la descripción de agregados obtenidos por métodos de aerosol facilita la comprensión de la tasa de colisión y sus morfologías [35]. Matsoukas y Friedlander (1991) propusieron la relación del volumen de los agregados y el radio de colisión, $r_{c}$, (ecuación 22),

$$
r_{c}=r^{p}\left(\frac{v}{v_{p}}\right)^{1 / D_{f}}=r_{p}\left(n_{p}\right)^{1 / D_{f}}
$$

Donde, $r^{p}$ es el radio de la partícula principal y $D_{f}$ la dimensión similar a un fractal de masa. La velocidad de coagulación, $\beta$, se calcula mediante la ecuación de Fuchs (23) propuesta en 1964, para las colisiones brownianas en el régimen libre molecular y continuo [35, 36].

$$
\beta=8 \pi D r_{c}\left[\frac{r_{c}}{2 r_{c}+\sqrt{2} g}+\frac{\sqrt{2} D}{c r_{c}}\right]^{-1}
$$

Donde $g$ es el parámetro de transición descrito entre las ecuaciones (24) y (26), $c$ es la velocidad de la partícula, $r_{c}$ es el radio de colisión y $D$ es el coeficiente de difusión de la partícula.

$$
\begin{aligned}
& g=\left[\frac{1}{6 r_{c} \frac{8 D}{\pi c}}\right]\left[\left(2 r_{c}+\frac{8 D}{\pi c}\right)^{3}-\left(4 r_{c}^{2}+\frac{8 D}{\pi c} 2\right)^{3 / 2}\right]-2 r_{c} \\
& c=\sqrt{\frac{8 K_{b} T}{\pi \rho_{p} v}} \\
& D=\frac{K_{b} T}{6 \pi r_{c} \mu}\left[\frac{5+4 \frac{\lambda}{r_{c}}+6\left(\frac{\lambda}{r_{c}}\right)^{2}+18\left(\frac{\lambda}{r_{c}}\right)^{3}}{5-\frac{\lambda}{r_{c}}+(8+\pi)\left(\frac{\lambda}{r_{c}}\right)^{2}}\right]
\end{aligned}
$$

\subsection{Condiciones del proceso de síntesis}

Durante la síntesis, las condiciones de procesamiento y sus efectos combinados, impactan las propiedades del material, permitiendo manipular las características de los productos [37, 38]. De ahí, que uno de los mayores desafíos de los investigadores es comprender la relación entre las condiciones de operación intrínsecas y extrínsecas y su influencia sobre propiedades de las partículas, obteniendo una visión integral del proceso [8], [13].

Los efectos de las condiciones del proceso suelen evaluarse como factores individuales, limitando el entendimiento de sus interacciones. La Tabla 2 recopila condiciones de síntesis de diferentes investigaciones donde se evaluaron algunos efectos sobre el tamaño de las partículas, mientras que la Figura 3 muestra la tendencia del tamaño de partícula y el área superficial específica de los productos a dichas condiciones. La variabilidad en los datos se debe al cambio de equipos y condiciones específicas de síntesis, sin embargo, es posible observar tendencias claras. 

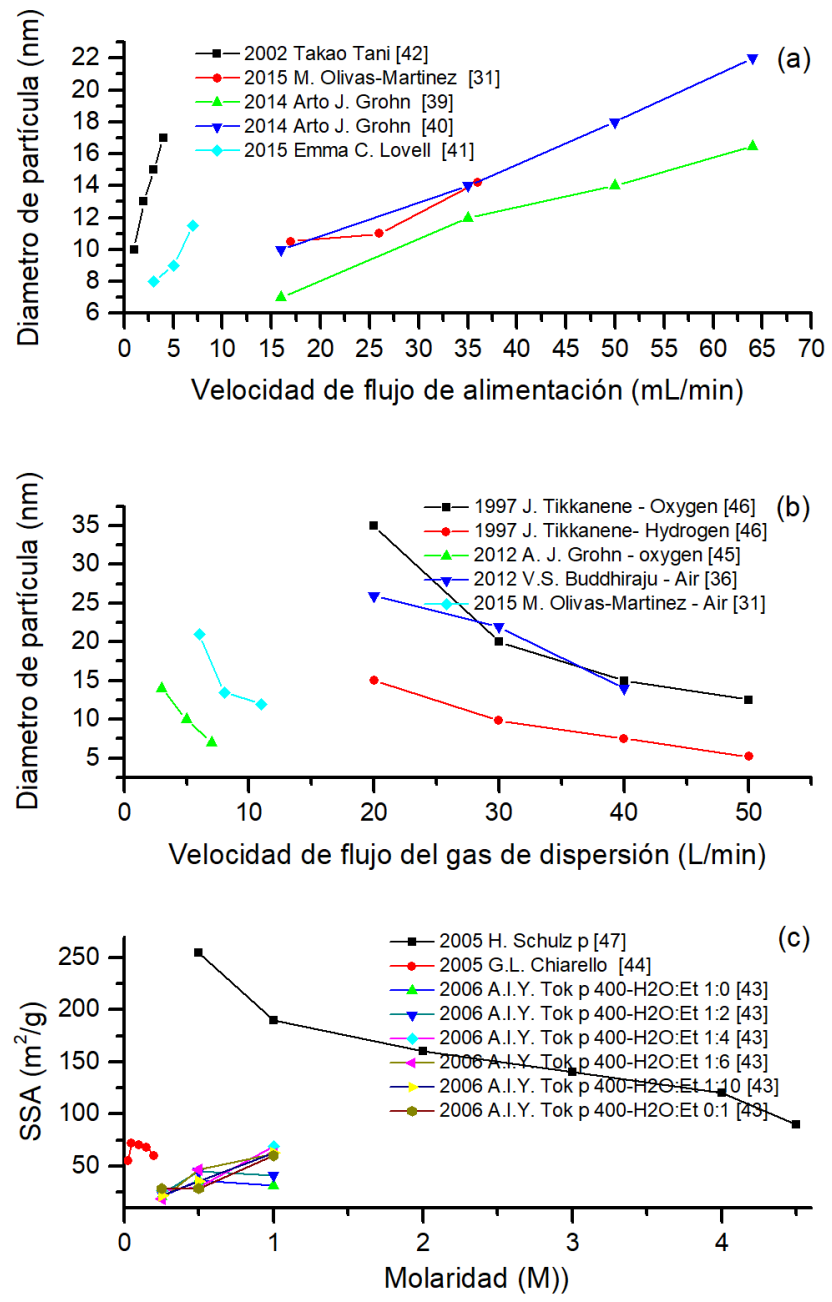

Figura 3: Efectos de las condiciones de síntesis sobre las partículas

La Figura 3-a muestra el incremento del tamaño de partícula, al aumentar la velocidad de flujo de alimentación. Durante el proceso, flujos de alimentación altos generan llamas largas debido al incremento de combustible e incrementan la concentración de iones aumentando el número de colisiones y coalescencia entre las partículas [39-42]. Por el contrario, aumentar la velocidad de flujo del gas de dispersión (Figura 3-b) disminuye la longitud de la llama, mejorando la mezcla de gases y favoreciendo la velocidad de consumo de las especies en fase gaseosa [31]. Estos fenómenos implican que la velocidad de combustión de las especies se rige por los parámetros del proceso y no por la cinética de reacción [31].

Durante la reacción, la vida útil de las gotitas se divide en dos etapas: calentamiento de las gotitas y evaporación. El calentamiento tarda $\sim 1 \mathrm{~ms}$, mientras que la tasa de evaporación aumenta al aumentar la presión del gas de dispersión, ya que los entornos turbulentos mejoran la velocidad de transferencia de calor [31].

La Figura 3-c relaciona la concentración de la solución y el área superficial específica, mostrando diferentes tendencias entre las investigaciones analizadas. Tok et al., (2006), reporta un aumento del SSA al incrementar la molaridad, atribuyendo este comportamiento a la alta temperatura generada por una alta tasa de disolvente en la llama. Sin embargo, al mismo tiempo los autores concuerdan en que una alta concentración incrementa la colisión de partículas disminuyendo el SSA, en contra de los resultados experimentales. Por otra parte, Chiarrello et al., (2005) y Schulz et al., (2005) muestran una disminución del SSA al aumentar la molaridad, debido a colisiones de partículas y procesos de sinterización [31, 41-44]. 
Por tanto, bajas tasas de flujo de alimentación o baja presión de dispersión aumentan el tiempo de residencia del aerosol en la llama favoreciendo el crecimiento de las partículas, al igual que el aumento de la concentración, incrementa el número de partículas primarias y el número de colisiones, promoviendo procesos de sinterización [41, 42].

Tabla 2: Condiciones de síntesis por FSP de las investigaciones analizadas.

\begin{tabular}{|c|c|c|c|c|c|c|}
\hline COMPUESTO & $\begin{array}{c}\text { PRECURSO- } \\
\text { RES/DISOLVENTE }\end{array}$ & CONCENTRACIÓN & $\begin{array}{l}\text { FLUJO DE } \\
\text { ALIMENTA- } \\
\text { CIÓN } \\
\text { (mL/min) }\end{array}$ & $\begin{array}{l}\text { GAS DE DIS- } \\
\text { PERSIÓN - FLU- } \\
\text { JO (L/min) }\end{array}$ & $\begin{array}{c}\text { COMBUSTI- } \\
\text { BLE/OXIDANTE } \\
\text { - FLUJO (L/min) }\end{array}$ & REREFENCIA \\
\hline $\mathrm{ZnO}$ & $\begin{array}{c}\mathrm{C}_{6} \mathrm{H}_{6} \mathrm{O}_{4} \mathrm{Zn} / \text { Metanol: } \\
\text { Ácido Acético }\end{array}$ & - & $1-4$ & Oxígeno & $\begin{array}{c}\text { Metano/ Oxígeno } \\
1,58 / 1,52 \\
\end{array}$ & TANI et al. [42] \\
\hline $\mathrm{SiO}_{2}$ & Organometálicos & - & $3 ; 5 ; 7$ & - & & $\begin{array}{c}\text { LOVELL et al. } \\
{[41]}\end{array}$ \\
\hline $\mathrm{SiO}_{2}$ & $\mathrm{SiC}_{8} \mathrm{H}_{20} \mathrm{O}_{4} /$ Etanol & $\begin{array}{l}50 \% \\
30 \%\end{array}$ & $\begin{array}{l}18,3 ; 26,2 \\
35,8 ; 26,2\end{array}$ & $\begin{array}{c}\text { Aire } \\
0,011 ; 0,006 \\
0,0083\end{array}$ & $\begin{array}{c}\text { Hidrogeno/ Oxí- } \\
\text { geno } \\
0,38 / 3,66\end{array}$ & $\begin{array}{c}\text { OLIVAS- } \\
\text { MARTINEZ et al. } \\
{[31]}\end{array}$ \\
\hline $\mathrm{ZrO}_{2}$ & $\mathrm{C}_{32} \mathrm{H}_{60} \mathrm{O}_{8} \mathrm{Zr} / \mathrm{Xilenos}$ & $1 \mathrm{M}$ & $16 ; 32 ; 64$ & $\begin{array}{c}\text { Oxígeno } \\
0,02 ; 0,04 ; 0,08\end{array}$ & $\begin{array}{c}\text { Metano/ Oxígeno } \\
2 / 4,5\end{array}$ & $\begin{array}{l}\text { GRÖHN et al. } \\
\text { [39] }\end{array}$ \\
\hline $\mathrm{ZrO}_{2}$ & $\mathrm{C}_{32} \mathrm{H}_{60} \mathrm{O}_{8} \mathrm{Zr} / \mathrm{Xilenos}$ & $1 \mathrm{M}$ & 4 & Oxígeno & $\begin{array}{c}\text { Metano/ Oxígeno } \\
1,25 / 2,5\end{array}$ & $\begin{array}{c}\text { GRÖHN et al. } \\
\text { [40] }\end{array}$ \\
\hline $\mathrm{ZrO}_{2}$ & $\begin{array}{c}\mathrm{Zr}\left(\mathrm{OCH}_{2} \mathrm{CH}_{2} \mathrm{CH}_{3}\right)_{4} / \\
\text { Etanol-1-propanol }\end{array}$ & 0,$5 ; 1 \mathrm{M}$ & 4 & $\begin{array}{l}\text { Oxígeno } \\
3-7\end{array}$ & $\begin{array}{c}\text { Metano/ Oxígeno } \\
1,25 / 2,5\end{array}$ & $\begin{array}{c}\text { GRÖHN et al. } \\
{[45]}\end{array}$ \\
\hline $\mathrm{Al}_{2} \mathrm{O}_{3}$ & $\begin{array}{c}\mathrm{Al}\left(\mathrm{NO}_{3}\right)_{3} \cdot 9 \mathrm{H}_{2} \mathrm{O} / \mathrm{Isop} \\
\text { ropanol }\end{array}$ & - & 6,2 & $\begin{array}{l}\text { Helio } \\
20-50\end{array}$ & $\begin{array}{c}\text { Hidro- } \\
\text { geno/Oxígeno } \\
35 / 20\end{array}$ & $\begin{array}{c}\text { TIKKANEN et al. } \\
\text { [46] }\end{array}$ \\
\hline $\mathrm{SiO}_{2}$ & $\mathrm{SiC}_{8} \mathrm{H}_{20} \mathrm{O}_{4}$ & - & - & Aire & $\begin{array}{c}\text { Hidro- } \\
\text { geno/Oxígeno } \\
6 / 15\end{array}$ & $\begin{array}{c}\text { BUDDHIRAJU et } \\
\text { al. [36] }\end{array}$ \\
\hline $\mathrm{LaCoO}_{3}$ & $\begin{array}{c}\mathrm{La}\left(\mathrm{CH}_{3} \mathrm{COO}\right)_{3} \\
\mathrm{Co}\left(\mathrm{CH}_{3} \mathrm{COO}\right)_{2} / \text { Ácid } \\
\text { o propiónico }\end{array}$ & $0,025-0,2 \mathrm{M}$ & 1 & $\begin{array}{l}\text { Oxígeno } \\
5\end{array}$ & $\begin{array}{c}\text { Metano/ Oxígeno } \\
0,5 / 1\end{array}$ & $\begin{array}{c}\text { CHIARELLO et } \\
\text { al. }[44]\end{array}$ \\
\hline $\mathrm{Ta}_{2} \mathrm{O}_{5} / \mathrm{SiO}_{2}$ & $\begin{array}{c}\mathrm{Ta}\left(\mathrm{OC}_{4} \mathrm{H}_{9}\right)_{5}- \\
\mathrm{SiC}_{8} \mathrm{H}_{20} \mathrm{O}_{4} / \mathrm{Hexano}\end{array}$ & $0,5-4,5 \mathrm{M}$ & 5 & $\begin{array}{c}\text { Oxígeno /Aire } \\
5 \\
\end{array}$ & $\begin{array}{c}\text { Metano/ Oxígeno } \\
1,1 / 2,5 \\
\end{array}$ & $\begin{array}{c}\text { SCHULZ et al. } \\
\text { [47] }\end{array}$ \\
\hline $\mathrm{ZrO}_{2}$ & $\begin{array}{c}\mathrm{Zr}\left(\mathrm{NO}_{3}\right)_{4} / \mathrm{H}_{2} \mathrm{O}: \\
\text { etanol }\end{array}$ & $0,25-1 \mathrm{M}$ & - & $\begin{array}{l}\text { Gases de combus- } \\
\text { tión } \\
\end{array}$ & $\begin{array}{c}\text { Acetileno/Oxígeno } \\
65 / 70 \mathrm{psi}\end{array}$ & TOK et al. [43] \\
\hline
\end{tabular}

\section{EQUIPO DE SÍNTESIS}

El equipo de síntesis se puede dividir en: sistema de atomización, zona de reacción y sistema de recolección (Figura 4).

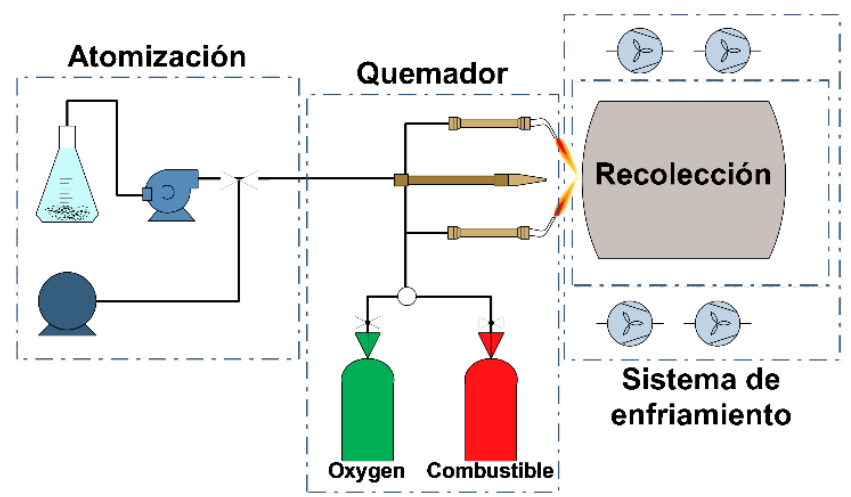

Figura 4: Esquema del proceso en FSP 


\subsection{Sistema de atomización}

La formación del aerosol es una etapa crítica al usar precursores de baja volatilidad, de lo contrario su influencia disminuye [48]. La uniformidad de las gotas de aerosol, velocidad de caída y distribución de tamaño depende de las propiedades de la solución, la geometría de la boquilla y el medio gaseoso [48]. La atomización consiste en la desintegración del líquido en pequeñas gotas cuando las fuerzas de tensión superficial del líquido son superadas por una fase gaseosa circundante con alta energía cinética, incrementando el área superficial específica del líquido [49].

El tamaño de gota describe la calidad del aerosol, el cual se puede expresar mediante la ecuación del diámetro medio de Sauter (SMD) al ser la más apropiada en procesos de aerosol con transferencia de masa y transferencia de calor [50]. La ecuación empírica de SMD (27) descrita por Nukiyama y Tanasawa para las boquillas de aire comprimido con un orificio máximo de $2 \mathrm{~mm}$ [51], está formada por dos términos, dominados, velocidad del aire y viscosidad [46, 52], donde el aerosol es afectado por la densidad, viscosidad y fuerzas de tensión superficial de las fases líquido/gas [49].

$$
S M D=\frac{0.585}{U_{R}}\left(\frac{\sigma_{L}}{\rho_{L}}\right)^{0.5}+53\left(\frac{\mu_{L}^{2}}{\sigma_{L} \rho_{L}}\right)^{0.225}\left(\frac{Q_{L}}{Q_{A}}\right)^{1.5}
$$

Donde $U_{R}$ es la velocidad relativa de aire al líquido, $\sigma_{\mathrm{L}}$ es la tensión superficial del líquido, $\rho_{\mathrm{L}}$ es la densidad del líquido, $\mu_{\mathrm{L}}$ es la viscosidad del líquido y $\mathrm{Q}_{\mathrm{L}}$ y $\mathrm{Q}_{\mathrm{A}}$ son la tasa de flujo volumétrico del líquido y el aire, respectivamente.

Un aerosol puede definirse en términos del incremento del área superficial del líquido después de la atomización. El área superficial antes de la atomización es el área del cilindro de líquido que sale por la boquilla. Después de la atomización, esta área es la suma del área superficial de cada gota. La multiplicación de estos factores indica el nivel de atomización alcanzado. Por otro lado, la tensión superficial del líquido representa la resistencia del líquido para formar una nueva área superficial. Así, la energía mínima requerida durante la atomización equivale a la tensión superficial multiplicada por el incremento del área superficial del líquido [49].

Las boquillas de inyección de aire son atomizadores ampliamente usados, formados por dos tubos concéntricos donde la velocidad del líquido $(<100 \mathrm{~m} / \mathrm{s})$ suele ser menor que la velocidad del aire, por lo que el tamaño de las gotas es menos sensible a las variaciones de viscosidad y densidad del líquido. La característica más importante de esta boquilla es el diámetro del orificio externo, donde el diámetro medio de gota es proporcional a su raíz cuadrada [49].

La velocidad de aspersión depende del flujo de alimentación de la solución y la presión del gas de dispersión. Estos factores afectan el tamaño de la gota, el cual determina la velocidad de evaporación y tiempo de residencia en la zona de reacción. Al incrementar el flujo de gas de dispersión, el cilindro liquido se rompe más fácilmente y el tiempo de residencia en la llama disminuye, formando pequeñas partículas primarias. Por otro lado, los efectos del flujo de alimentación de la solución de partida no son claros. OLIVAS et al. [31], presenta un modelo que predice la disminución de tamaño de partícula al aumentar el flujo de alimentación, debido al aumento de los puntos de nucleación que favorecen la formación de nanopartículas. Por otra parte, H. CHANG et al., M. AROMAA et al. y LOVELL et al. [11, 41, 53] indican que su incremento conduce a incrementos en el tamaño de partícula, debido a una alta concentración de partículas en la zona de alta temperatura favoreciendo procesos de coalescencia. A. PURWANTO et al., [54], predice el tamaño de partícula teniendo en cuenta las propiedades físicas del precursor, de modo que el diámetro de la partícula está dado por (28),

$$
d_{p}=\left[\frac{C_{D} d_{D}^{3} \cdot M W}{n \cdot \rho}\right]^{1 / 3}
$$

Donde $C_{D}$ es la concentración de la gota, $d_{D}$ el diámetro de la gota, $M W$ el peso molecular de las partículas, $n$ la relación estequiométrica y $\rho$ la densidad de las partículas. [54]. Considerando esta ecuación, el diámetro de partícula es proporcional a la concentración y diámetro de las gotas, en coherencia con $\mathrm{H}$. CHANG et al. [11], M. AROMAA et al [53]. y LOVELL et al. [41].

\subsection{Zona de reacción}

En la zona de reacción, la llama puede ser expuesta al aire o encerrada [25]. Las llamas cerradas presentan menores pérdidas de calor por convección y radiación, controlando mejor las condiciones del proceso, sin 
embargo, favorecen el crecimiento de las partículas, al contrario de los sistemas al aire libre [55].

Los quemadores de llama por difusión de flujo, como el quemador de múltiples anillos, formado por tres tubos concéntricos de acero inoxidable, son ampliamente usados en la industria (Figura 5). La configuración clásica (b) usa el tubo externo para el oxidante, el tubo medio para el combustible y el interno para la mezcla de precursor/gas de dispersión, mientras otras, ubican el combustible en el tubo más externo y el oxidante en el tubo medio (c), mostrando mayor potencial para obtener partículas finas, pasando de $105 \mathrm{~nm}$ a 11 $\mathrm{nm}[19,56,57]$.

Las llamas premezcladas promueven una alta concentración de radicales formados durante las reacciones de oxidación de los precursores, sin afectar la disposición de especies para óxidos mixtos [19]. Algunos quemadores de llamas premezcladas son el quemador McKenna (d), el cual produce llamas planas premezcladas, con una distribución uniforme de la temperatura en una geometría simple [58] y el quemador de Doble llama (e), en el que dos llamas individuales premezcladas colisionan, formando un punto medio con alta temperatura, donde el aerosol se descompone [17, 59].

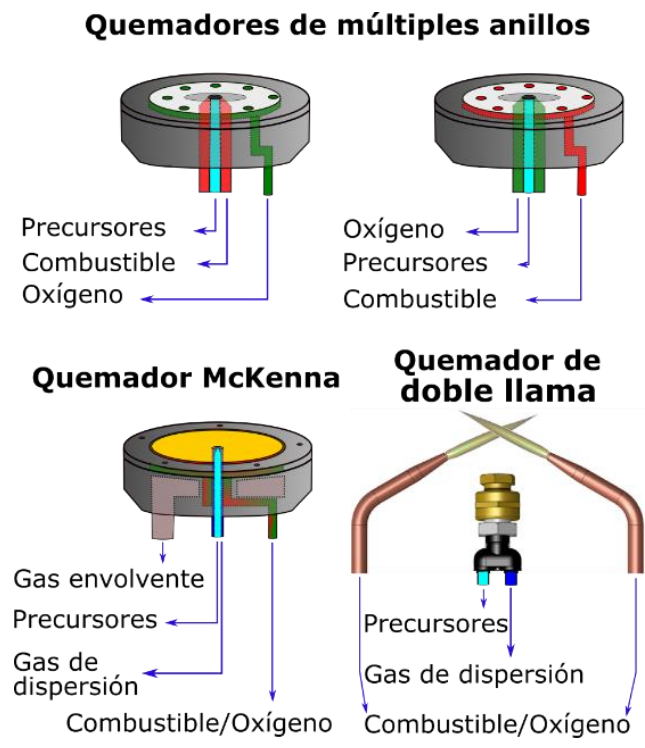

Figura 5: Quemadores más comunes en procesos de síntesis por FSP

Los combustibles de mayor uso son metano, hidrógeno y acetileno, los cuales ofrecen diferentes ambientes de combustión y energía a la reacción [46]. El hidrógeno permite la formación de materiales muy puros ya que su combustión no produce radicales de carbono [46]; sin embargo su instalación es compleja. Por otro lado, el acetileno es un gas económico que proporciona mayor entalpía de combustión que otros combustibles, siendo un excelente candidato como gas de combustión [60].

\subsection{Recolección de las partículas}

Como sistemas de recolección de partículas son usadas cámaras de filtro, precipitadores electrostáticos, precipitadores termoforéticos y ciclones, que ofrecen diferentes valores de rendimiento al proceso de recolección. De ellos, los precipitadores electrostáticos ofrecen excelentes resultados, al tener una eficiencia por encima del $65 \%$ y evitar el continuo recambio de filtros.

Los precipitadores electrostáticos (EPS) se componen de dos superficies metálicas, entre las cuales se forma un campo electrostático debido a la acción de un electrodo negativo o de descarga y un electrodo positivo o colector. Estos equipos son usualmente de cobre o aluminio debido a su buena conductividad eléctrica, presentando diferentes diseños según la necesidad de la industria. Entre los diseños más usados se encuentran los precipitadores tubulares o cilindros coaxiales, de placas paralelas y placa-alambre [61, 62].

En estos sistemas un campo eléctrico carga las partículas, viéndose afectadas por los iones de gas y el movimiento Browniano aleatorio de iones negativos y sus colisiones con pequeñas partículas [63]. Para iniciar una reacción, este sistema se precalienta evaporando etanol en la llama, evitando la condensación de agua dentro del sistema [64] y finalmente recolectar las partículas en el precipitador [65]. Durante la recolección, las partículas continúan experimentando fenómenos de crecimiento. Por esa razón, los colectores generalmente están equipados con un sistema de enfriamiento para disminuir la temperatura del recolector, evitar 
procesos de sinterización [48] y controlar los fenómenos de crecimiento [19].

La eficiencia del precipitador depende del área de recolección total y la diferencia de potencial que genera el campo eléctrico entre dos placas metálicas. La eficiencia del precipitador está determinada por la ecuación de Deutsch-Anderson, que se muestra en la ecuación (29).

$$
\eta=1-\mathrm{e}^{\left(-\frac{V_{p m} A_{c}}{Q}\right)}
$$

Donde $\eta$ es la eficiencia de recolección del precipitador, $A c$ es el área de recolección efectiva $\left(\mathrm{m}^{2}\right), V p m$ es la velocidad de migración de partículas $(\mathrm{m} / \mathrm{s})$ y $Q$ es el caudal volumétrico de gas $\left(\mathrm{m}^{3} / \mathrm{s}\right)$. Esta ecuación no considera el tamaño de partícula y la distribución de partículas de los polvos, asumiendo una velocidad de migración uniforme, ignorando que las partículas más grandes tienen una mayor velocidad de migración que las partículas más pequeñas. Además, no se contempla la deposición de partículas en la superficie del precipitador o el factor de fuga a través del sistema. Por otra parte, la velocidad de migración de las partículas en la ecuación de Deutsch-Anderson depende de las fuerzas principales que actúan en la partícula, correspondientes a las fuerzas gravitacionales, eléctricas, viscosas e inerciales. Por lo que, la ecuación de velocidad de migración de partículas se expresa como (30),

$$
V_{p m}=\frac{q E C u}{3 \pi \mu d_{p}}
$$

Donde, $q$ corresponde a la carga de la partícula, $\mathrm{Cu}$ es el factor de corrección de Cunningham, $E$ es el campo eléctrico del colector, $\mu$ es la viscosidad y $d$ es el tamaño de partícula. A partir de las ecuaciones (29) y (30) es posible observar que mayores caudales volumétricos $(Q)$ y pequeños tamaños de partículas conducen a menores eficiencias en el precipitador. Además, una mayor área de recolección aumenta la eficiencia, pero aumenta los costos de producción y dificulta el proceso de limpieza [62, 66].

\section{CONCLUSIONES, RETOS Y FUTURAS INVESTIGACIONES}

Pirólisis de aerosol en llama es un método eficiente para la producción de nanopartículas en continuo, el cual ha tenido grandes avances en etapas de escalamiento. Durante la síntesis de nanopartículas, los fenómenos físicos y químicos involucrados en los procesos de evaporación, descomposición, reacción y crecimiento, son estudiados mediante el uso de modelos matemáticos que posibilitan la manipulación del proceso para diseñar nanomateriales. No obstante, el desarrollo de modelos sigue siendo un tema de estudio e interés, debido a la complejidad del proceso, al involucrar fenómenos de combustión y formación de nanopartículas de forma conjunta.

El potencial para desarrollar nuevos materiales es evidente, debido a la facilidad de transformación de precursores organometálicos en compuestos cerámicos, sin embargo, esto también implica grandes retos en el entendimiento de la influencia de los sistemas químicos sobre las características y propiedades de los productos. El uso de compuestos metalorgánicos y organometálicos, permite la formulación de soluciones estables, la evaporación de los precursores metálicos antes de la evaporación del disolvente y la generación de reacciones en fase gaseosa que conducen a la formación de nanopartículas.

De igual forma, el diseño del equipo y las condiciones de síntesis afectan las características de los productos, por lo cual el estudio de parámetros operacionales como el tipo de precursores, su concentración y flujo de alimentación, el flujo del gas de dispersión y los combustibles suponen grandes avances en el entendimiento de los mecanismos de formación de gotas, su evaporación y posterior transformación en nanopartículas. De esta forma, factores como el tamaño de gota, que describen la calidad del aerosol, determinan la velocidad de evaporación y el tiempo de residencia en la reacción y por tanto el mecanismo de formación gota-partícula ó gas-partícula, se ven afectados por la velocidad de flujo de alimentación de la solución de partida y la presión del gas de dispersión.

Sobre los parámetros operacionales más estudiados se puede concluir que el tamaño de partícula aumenta al usar baja presión de dispersión que aumente el tiempo de residencia del aerosol en la llama, altos 
flujos de alimentación que aumenten significativamente la temperatura de la zona de reacción debido al incremento de combustible alimentado en forma de disolvente, y altas concentraciones de precursores que aumenten la concentración de partículas primarias en el ambiente de reacción, aumentando el número de colisiones que promueven la sinterización de las partículas. Sin embargo, el proceso involucra otros parámetros como tipo de disolventes, combustibles, presiones de descarga, velocidad de enfriamiento, entre otros, que también afectan el proceso y requieren mayor estudio.

\section{AGRADECIMIENTOS}

Este trabajo fue apoyado por el Departamento Administrativo de Ciencia, Tecnología e Innovación (COLCIENCIAS) llamado 647 de 2014, Fundación para la Promoción de la Investigación y la Tecnología (FPIT) Banco de la República - Número de contrato 201716 y Grupo de Investigación de Cemento y Materiales de Construcción de la Universidad Nacional de Colombia.

\section{BIBLIOGRAFÍA}

[1] SOKOLOWSKI, M., SOKOLOWSKA, A., MICHALSKI, A., et al. "The 'In-flame-reaction' methos for Al2O3 aerosol formation," J. Aerosol Sci., v. 8, n. 1946, 1977.

[2] LAINE, R.M., BICKMORE, C.R., TREADWELL, D.R., et al., "Ultrafine metal oxide powders by Flame Spray Pyrolysis," 5958361, 1999.

[3] LAINE, R.M., RAND, S.C., HINKLIN, T., et al., "Ultrafine powders and their use as lasing media," 6656588 B1, 2003.

[4] SUTORIK, A.C., LAINE, R.M., MARCHAL, J., et al., "Mixed-metal oxide particles by Liquid Feed Flame Spray Pyrolysis of oxide precursors in oxigenated solvents,” 7220398, 2007.

[5] STROBEL, R., BAIKER, A., PRATSINIS, S.E. "Aerosol flame synthesis of catalysts," Adv. Powder Technol., v. 17, n. 5, pp. 457-480, Aug. 2006.

[6] ALEMÁN, J., et al., "Definitions of terms relating to the structure and processing of sols, gels, networks, and inorganic-organic hybrid materials (IUPAC recommendations 2007)," Pure Appl. Chem., v. 79, n. 10, pp. 1801-1829, 2007.

[7] MEIERHOFER, F., MÄDLER, L., FRITSCHING, U. "Nanoparticle evolution in flame spray pyrolysisProcess design via experimental and computational analysis," AIChE J., v. 66, n. 2, pp. 1-14, 2020.

[8] SCHULZ, C., DREIER, T., FIKRI, M., et al., "Gas-phase synthesis of functional nanomaterials: Challenges to kinetics, diagnostics, and process development," Proc. Combust. Inst., vol. 37, no. 1, pp. 83$108,2019$.

[9] ABDELSAMIE, A., KRUIS, F.E., WIGGERS, H., et al., "Nanoparticle Formation and Behavior in Turbulent Spray Flames Investigated by DNS,” Flow, Turbul. Combust., n. 0123456789, 2020.

[10] MÄDLER, L., "Liquid-fed Aerosol Reactors for One-step Synthesis of Nano-structured Particles †," Kona, v. 22, n. 22, pp. 107-120, 2004.

[11] CHANG, H., PARK, J.-H., JANG, H.D. "Flame synthesis of silica nanoparticles by adopting two-fluid nozzle spray," Colloids Surfaces A Physicochem. Eng. Asp., v. 313-314, pp. 140-144, Feb. 2008.

[12] PRATSINIS, S.E. "Flame synthesis of nanosize particles: Precise control of particle size," J. Aerosol Sci., v. 27, n. 96, pp. S153-S154, Sep. 1996.

[13] TEOH, W.Y., AMAL, R., MÄDLER, L. "Flame spray pyrolysis: An enabling technology for nanoparticles design and fabrication.," Nanoscale, v. 2, n. 8, pp. 1324-1347, 2010.

[14] TEOH, W. "A Perspective on the Flame Spray Synthesis of Photocatalyst Nanoparticles," Materials (Basel)., v. 6, n. 8, pp. 3194-3212, Jul. 2013.

[15] KIM, M., LAI, S., LAINE, R.M. “Combinatorial Nanopowder Synthesis Along the ZnO-Al2O3 Tie Line Using Liquid-Feed Flame Spray Pyrolysis,” J. Am. Ceram. Soc., v. 94, n. 10, pp. 3308-3318, Oct. 2011.

[16] HINKLIN, T., et al., "Liquid-Feed Flame Spray Pyrolysis of Metalloorganic and Inorganic Alumina Sources in the Production of Nanoalumina Powders," Chem. Mater, v. 16, n. 12, pp. 21-30, 2004.

[17] AZURDIA, J., et al., "Liquid-Feed Flame Spray Pyrolysis as a Method of Producing Mixed-Metal Oxide Nanopowders of Potential Interest as Catalytic Materials . Nanopowders along the NiO-Al 2 O 3 Tie 
Line Including," Chem. Mater., n. 6, pp. 731-739, 2006.

[18] MOLINA, P., BARBEITO, P., ALABART, G. "Preparación de precursores metalorgánico anhidridos y su uso para la deposición y crecimiento de capas y cintas superconductoras," 2259919, 2006.

[19] LI, S., REN, Y., BISWAS, P., et al., "Flame aerosol synthesis of nanostructured materials and functional devices: Processing, modeling, and diagnostics," Prog. Energy Combust. Sci., v. 55, pp. 1-59, 2016.

[20] WOOLDRIDGE, M.S. "Gas-phase combustion synthesis," Prog. Energy Combust. Sci., v. 24, pp. $63-$ 87, 1998.

[21] PRATSINIS, S.E. "Flame aerosol synthesis of ceramic powders," Prog. Energy Combust. Sci., v. 24, n. 3, pp. 197-219, 1998.

[22] BAD SODEN, S.K., KARLSTEIN, P.K., KAHL, H.A., et al."Process for producing metal oxide powders by means of flame spray pyrolysis," 2019.

[23] JOSSEN, R., PRATSINIS, S.E., STARK, W.J., et al., "Criteria for flame-spray synthesis of hollow, shell-like, or inhomogeneous oxides," J. Am. Ceram. Soc., v. 88, n. 6, pp. 1388-1393, 2005.

[24] GUOZHONG, C. Nanostructures \& Nanomaterials- Synthesis, Properties \& Applications, World Scie. University od Washington, USA: Imperial College Press, 2004.

[25] WASER, O., GROEHN, A.J., EGGERSDORFER, M.L., et al., "Air Entrainment During Flame Aerosol Synthesis of Nanoparticles," Aerosol Sci. Technol., v. 48, n. 11, pp. 1195-1206, 2014.

[26] CAMENZIND, A., CASERI, W.R., PRATSINIS, S.E. "Flame-made nanoparticles for nanocomposites," Nano Today, v. 5, n. 1, pp. 48-65, 2010.

[27] MÄDLER, L., KAMMLER, H.K., MUELLER, R., et al., "Controlled synthesis of nanostructured particles by Flame spray pyrolysis," J. Aerosol Sci., v. 33, pp. 369-389, 2002.

[28] ULRICH, G.D. "Theory of Particle Formation and Growth in Oxide Synthesis Flames Theory of Particle Formation and Growth in Oxide Synthesis Flames," Combust. Sci. Technol., v. 4, n. 1, pp. 47-57, 1971.

[29] ROTH, P. "Particle Synthesis in Flames Keywords," pp. 1-43, 2006.

[30] BETANCUR-GRANADOS, N., RESTREPO, J.C., TOBÓN, J.I., et al., "Dicalcium silicate (2CaO·SiO2) synthesized through flame spray pyrolysis and solution combustion synthesis methods," Ceram. Int., v. 45, n. July, pp. 9589-9595, 2019.

[31] OLIVAS-MARTINEZ, M., SOHN, H.Y., JANG, H.D., et al., "Computational fluid dynamic modeling of the flame spray pyrolysis for silica nanopowder synthesis," J. Nanopart Res, v. 17, n. 324, 2015.

[32] CHEN, H., MULMUDI, H.K., TRICOLI, A. "Flame spray pyrolysis for the one-step fabrication of transition metal oxide films: Recent progress in electrochemical and photoelectrochemical water splitting," Chinese Chem. Lett., v. 31, n. 3, pp. 601-604, 2020.

[33] GUTSCH, A., MÜHLENWEG, H., KRÄMER, M. "Tailor-made nanoparticles via gas-phase synthesis," Small, v. 1, n. 1, pp. 30-46, 2005.

[34] KOCH, W., FRIEDLANDER, S.K. "The effect of particle coalescence on the surface area of a coagulating aerosol," J. Colloid Interface Sci., v. 140, n. 2, pp. 419-427, 1990.

[35] KRUIS, F.E.., KUSTERS, K.A., PRATSINIS, S.E., et al., "A Simple Model for the Evolution of the Characteristics of Aggregate Particles Undergoing Coagulation and Sintering," Aerosol Sci. Technol., vol. 19, no. 4, pp. 514-526, 1993.

[36] BUDDHIRAJU, V.S., RUNKANA, V. "Simulation of nanoparticle synthesis in an aerosol flame reactor using a coupled flame dynamics-monodisperse population balance model," J. Aerosol Sci., vol. 43, no. 1, pp. 1-13, Jan. 2012.

[37] LEE, S., SCHNEIDER, K., SCHUMANN, J., et al., "Effect of metal precursor on $\mathrm{Cu} / \mathrm{ZnO} / \mathrm{Al} 2 \mathrm{O} 3$ synthesized by flame spray pyrolysis for direct DME production," Chem. Eng. Sci., v. 138, pp. 194-202, 2015.

[38] BETANCUR-GRANADOS, N., RESTREPO-BAENA, O.J. "Flame spray pyrolysis synthesis of ceramic nanopigments CoCr2O4: The effect of key variables," J. Eur. Ceram. Soc., 2017.

[39] GRÖHN, A.J., PRATSINIS, S.E., WEGNER, K. "Scale-up for Nanoparticle Synthesis by Flame Spray Pyrolysis : The High Temperature Particle Residence Time,” Ind. Eng. Chem. Res., v. 53, pp. 10734-10742, 2014.

[40] GRÖHN, A.J., EGGERSDORFER, M.L., PRATSINIS, S.E., et al., "On-line monitoring of primary and agglomerate particle dynamics," J. Aerosol Sci., v. 73, pp. 1-13, 2014. 
[41] LOVELl, E., SCOTT, J., AMAL, R. "Ni-SiO2 Catalysts for the Carbon Dioxide Reforming of Methane: Varying Support Properties by Flame Spray Pyrolysis," Molecules, v. 20, n. 3, pp. 4594-4609, 2015.

[42] TANI, T., MADLER, L., PRATSINIS, S.E. "Homogeneous ZnO nanoparticles by flame spray pyrolysis," J. nan, pp. 337-343, 2002.

[43] TOK, A.I., BOEY, F.Y., DU, S., et al., "Flame spray synthesis of $\mathrm{ZrO} 2$ nano-particles using liquid precursors," Mater. Sci. Eng. B, v. 130, n. 1-3, pp. 114-119, Jun. 2006.

[44] CHIARELLO, G., ROSSETTI, I., FORNI, L. "Flame-spray pyrolysis preparation of perovskites for methane catalytic combustion," J. Catal., v. 236, n. 2, pp. 251-261, Dec. 2005.

[45] GRÖHN, A.J., PRATSINIS, S.E., WEGNER, K. "Fluid-particle dynamics during combustion spray aerosol synthesis of ZrO2," Chem. Eng. J., v. 191, pp. 491-502, 2012.

[46] TIKKANEN, J. et al., "Characteristics of the liquid flame spray process," Surf. Coat. Technol., v. 90, pp. 210-216, 1997.

[47] SCHULZ, H., MÄDLER, L., PRATSINIS, S.E., et al., "Transparent nanocomposites of radiopaque, flame-made Ta2O 5/SiO2 particles in an acrylic matrix," Adv. Funct. Mater., v. 15, n. 5, pp. 830-837, 2005.

[48] TROMMER, R.M., BERGMANN, C.P. Flame Spray Technology - Method for production of nanoparticles. Springer, 2015.

[49] LEFEBVRE, A., MCDONELL, V. Atomization and Sprays, Second Edition. 2017.

[50] LEFEBVRE, A.H. “Airblast atomization,” Prog. Energy Combust. Sci., v. 6, pp. 233-261, 1980.

[51] NUKIYAMA, S., TANASAWA, Y. "Experiments on the atomization of liquids in an air stream," 1950.

[52] HEINE, M.C., MÄDLER, L., JOSSEN, R., et al., "Direct measurement of entrainment during nanoparticle synthesis in spray flames," Combust. Flame, v. 144, n. 4, pp. 809-820, Mar. 2006.

[53] AROMAA, M., KESKINEN, H., MÄKELÄ, J. "The effect of process parameters on the Liquid Flame Spray generated titania nanoparticles.," Biomol. Eng., v. 24, n. 5, pp. 543-8, Nov. 2007.

[54] ASHGRIZ, N. Handbook of Atomization and Sprays. Springer Netherlands, 2011.

[55] WEI, J., REN, Y., ZHANG, Y., et al., "Effects of temperature-time history on the flame synthesis of nanoparticles in a swirl-stabilized tubular burner with two feeding modes," J. Aerosol Sci., v. 133, n. November 2018, pp. 72-82, 2019.

[56] ALSULAMI, R., WINDELL, B., NATES, S., et al., "Investigating the role of atomization on flame stability of liquid fuels in an annular spray burner," Fuel, v. 265, n. October 2019, p. 116945, 2020.

[57] SCHNEIDER, F., et al., "SpraySyn-A standardized burner configuration for nanoparticle synthesis in spray flames,” Rev. Sci. Instrum., v. 90, n. 8, 2019.

[58] MIGLIORINI, F., DE IULIIS, S., CIGNOLI, F., et al., "How 'flat' is the rich premixed flame produced by your McKenna burner?," Combust. Flame, v. 153, n. 3, pp. 384-393, 2008.

[59] BETANCUR-GRANADOS, N. "Synthesis of calcium silicates by flame spray pyrolysis," Universidad Nacional de Colombia, 2019.

[60] RUDIN, T., WEGNER, K., PRATSINIS, S.E. “Uniform nanoparticles by flame-assisted spray pyrolysis (FASP) of low cost precursors,” J. Nanoparticle Res., v. 13, n. 7, pp. 2715-2725, 2011.

[61] RODRÍGUEZ, J.F., ALARCÓN PINTO, U.F., Garcia Bedoya, O., et al., "Diseño y prototipo de un precipitador electrostático," Rev. Mutis, v. 7, n. 2, pp. 86-95, 2017.

[62] CHANG, J.-S., KELLY, A.J., CROWLEY, J.M. Handbook of electrostatic processes, v. 53, n. 9. New York: Marcel Dekker, Inc., 1995.

[63] PATIÑO, D., CRESPO, B., PORTEIRO, J., et al., "Experimental study of a tubular-type ESP for smallscale biomass boilers: Preliminary results in a diesel engine," Powder Technol., v. 288, n. November 2015, pp. 164-175, 2016.

[64] BICKMORE, C.R., WALDNER, K.F., TREADWELL, D.R., et al., "Ultrafine Spinel Powders by flame Spray Pyrolysis of a Magnesium Aluminum Double Alkoxide.” 1996.

[65] BÍCKMORE, C.R., WALDNER, K.F., TREADWEN, D.R., et al., "Ultrafine Spinel Powders by Flame Spray Pyrolysis of a Magnesium Aluminium Double Alkoxide," J. Am. Ceram. Soc., v. 3, n. 2, pp. 14191423, 1996.

[66] PARKER, K.R. Applied electrostatic precipitation. 1997. 


\section{ORCID}

Natalia Betancur-Granados

Jorge Iván Tobón

Oscar Jaime Restrepo-Baena https://orcid.org/0000-0003-3281-2946

https://orcid.org/0000-0002-1451-1309

https://orcid.org/0000-0003-3944-9369 